\title{
Front Matter: Volume 8431
}

, "Front Matter: Volume 8431," Proc. SPIE 8431, Silicon Photonics and Photonic Integrated Circuits III, 843101 (24 May 2012); doi:

10.1117/12.979312

SPIE. Event: SPIE Photonics Europe, 2012, Brussels, Belgium 


\title{
PROCEEDINGS OF SPIE
}

\section{Silicon Photonics and Photonic Integrated Circuits III}

\author{
Laurent Vivien \\ Seppo K. Honkanen \\ Lorenzo Pavesi \\ Stefano Pelli \\ Editors
}

16-19 April 2012

Brussels, Belgium

Sponsored by

SPIE

Cosponsored by

B-PHOT-Brussels Photonics Team (Belgium)

Brussels-Capital Region (Belgium)

FWO_Fonds Wetenschappelijk Onderzoek (Belgium)

ICO-International Commission for Optics

Ville de Bruxelles (Belgium)

Cooperating Organisations

$\mathrm{CBO}-\mathrm{BCO}$ (Belgium)

EOS-European Optical Society (Germany)

IET-The Institution of Engineering and Technology (United Kingdom)

IOP_Institute of Physics (United Kingdom)

Photonics4Life (Germany)

Photonics@be (Belgium)

Photonics 21 (Germany)

PromOptica (Belgium)

Published by

SPIE

Volume 8431

Proceedings of SPIE, 0277-786X, v. 8431 
The papers included in this volume were part of the technical conference cited on the cover and title page. Papers were selected and subject to review by the editors and conference program committee. Some conference presentations may not be available for publication. The papers published in these proceedings reflect the work and thoughts of the authors and are published herein as submitted. The publisher is not responsible for the validity of the information or for any outcomes resulting from reliance thereon.

Please use the following format to cite material from this book:

Author(s), "Title of Paper," in Silicon Photonics and Photonic Integrated Circuits III, edited by Laurent Vivien, Seppo K. Honkanen, Lorenzo Pavesi, Stefano Pelli, Proceedings of SPIE Vol. 8431 (SPIE, Bellingham, WA, 2012) Article CID Number.

ISSN 0277-786X

ISBN 9780819491237

Published by

SPIE

P.O. Box 10, Bellingham, Washington 98227-0010 USA

Telephone +1 3606763290 (Pacific Time) · Fax +1 3606471445

SPIE.org

Copyright (C) 2012, Society of Photo-Optical Instrumentation Engineers

Copying of material in this book for internal or personal use, or for the internal or personal use of specific clients, beyond the fair use provisions granted by the U.S. Copyright Law is authorized by SPIE subject to payment of copying fees. The Transactional Reporting Service base fee for this volume is $\$ 18.00$ per article (or portion thereof), which should be paid directly to the Copyright Clearance Center (CCC), 222 Rosewood Drive, Danvers, MA 01923. Payment may also be made electronically through CCC Online at copyright.com. Other copying for republication, resale, advertising or promotion, or any form of systematic or multiple reproduction of any material in this book is prohibited except with permission in writing from the publisher. The CCC fee code is 0277-786X/12/\$18.00.

Printed in the United States of America.

Publication of record for individual papers is online in the SPIE Digital Library.

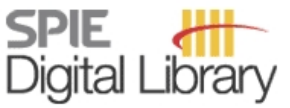

SPIEDigitalLibrary.org

Paper Numbering: Proceedings of SPIE follow an e-First publication model, with papers published first online and then in print and on CD-ROM. Papers are published as they are submitted and meet publication criteria. A unique, consistent, permanent citation identifier (CID) number is assigned to each article at the time of the first publication. Utilization of CIDs allows articles to be fully citable as soon as they are published online, and connects the same identifier to all online, print, and electronic versions of the publication. SPIE uses a six-digit CID article numbering system in which:

- The first four digits correspond to the SPIE volume number.

- The last two digits indicate publication order within the volume using a Base 36 numbering system employing both numerals and letters. These two-number sets start with 00, 01, 02, 03, 04, $05,06,07,08,09,0 A, 0 B \ldots 0 Z$, followed by 10-1Z, 20-2Z, etc.

The CID number appears on each page of the manuscript. The complete citation is used on the first page, and an abbreviated version on subsequent pages. Numbers in the index correspond to the last two digits of the six-digit CID number. 


\section{Contents}

xi Conference Committee
xiii Introduction

NONLINEAR INTEGRATED PHOTONICS

843104 Silicon on sapphire and SOI photonic devices for mid-infrared and near-IR wavelengths (Invited Paper) [8431-03]

O. Boyraz, Univ. of California, Irvine (United States) and Istanbul Sehir Univ. (Turkey); Y. Huang, Univ. of California, Irvine (United States); X. Sang, Beijing Univ. of Posts and Telecommunications (China)

843106 Efficient four-wave-mixing-based wavelength conversion in silicon nanowire rings without dispersion engineering [8431-05]

N. Vermeulen, Vrije Univ. Brussel (Belgium); J. E. Sipe, Univ. of Toronto (Canada); Y. Lefevre, C. Debaes, H. Thienpont, Vrije Univ. Brussel (Belgium)

NONLINEAR OPTICS

843108 Energy efficient all-optical signal processing in SOI: nanowires or slow-light structures? [8431-07]

C. Husko, B. J. Eggleton, The Univ. of Sydney (Australia)

\section{PHOTODETECTORS}

8431 OA 40Gbit/s germanium waveguide photodetector on silicon [8431-09]

L. Virot, Institut d'Electronique Fondamentale, CNRS, Univ. Paris-Sud (France) and CEA-LETI-Minatec (France) and STMicroelectronics (France); L. Vivien, Institut d'Electronique Fondamentale, CNRS, Univ. Paris-Sud (France); A. Polzer, Vienna Univ. of Technology (Austria); D. Marris-Morini, J. Osmond, Institut d'Electronique Fondamentale, CNRS, Univ. Paris-Sud (France); J. M. Hartmann, CEA-LETI-Minatec (France); P. Crozat, E. Cassan, Institut d'Electronique Fondamentale, CNRS, Univ. Paris-Sud (France); C. Baudot, STMicroelectronics (France); C. Kopp, CEA-LETI-Minatec (France); F. Boeuf, STMicroelectronics (France); H. Zimmermann, Vienna Univ. of Technology (Austria); J. M. Fédéli, CEA-LETI-Minatec (France)

\section{LIGHT GUIDING AND COUPLING}

8431 OD Group IV photonic devices for the mid-infrared (Invited Paper) [8431-12]

G. Z. Mashanovich, M. Nedeljkovic, Univ. of Southampton (United Kingdom);

M. M. Milosevic, Univ. of Surrey (United Kingdom); Y. Hu, F. Y. Gardes, D. J. Thomson, T.-B. Masaud, E. Jaberansary, H. M. H. Chong, Univ. of Southampton (United Kingdom); R. Soref, Univ. of Massachusetts at Boston (United States); G. T. Reed, Univ. of Southampton (United Kingdom) 
8431 OE Grating couplers in thick rib SOI waveguides for TE and TM polarizations (Best Paper Award) [8431-13]

C. Alonso-Ramos, A. Ortega-Moñux, R. Halir, L. Zavargo-Peche, I. Molina-Fernández, Univ. de Málaga (Spain); P. Cheben, D.-X. XU, S. Janz, National Research Council Canada (Canada)

$8431 \mathrm{OF} \quad$ Reducing the temperature sensitivity of SOI waveguide-based biosensors [8431-61] K. B. Gylfason, Royal Institute of Technology (Sweden); A. Mola Romero, Royal Institute of Technology (Sweden) and Univ. of Barcelona (Spain); H. Sohlström, Royal Institute of Technology (Sweden)

$84310 G$ Design, fabrication, and characterisation of fully etched TM grating coupler for photonic integrated system-in-package [8431-15]

O. Gili-de-Villasante, P. Tcheg, Technische Univ. Berlin (Germany); B. Wang, A. Suna, Fraunhofer Institute for Reliability and Microintegration (Germany); G. Giannoulis, I. Lazarou, D. Apostolopoulos, H. Avramopoulos, National Technical Univ. of Athens (Greece); N. Pleros, Aristotle Univ. of Thessaloniki (Greece); M. Baus, M. Karl, AMO GmbH (Germany); T. Tekin, Technische Univ. Berlin (Germany) and Fraunhofer Institute for Reliability and Microintegration (Germany)

$8431 \mathrm{OH} \quad$ Fine tunable multi-cavity Si photonic crystal filters [8431-16] A. Baldycheva, Trinity College Dublin (Ireland); V. A. Tolmachev, Ioffe Physico-Technical Institute (Russian Federation); T. S. Perova, Trinity College Dublin (Ireland)

8431 Ol Narrow band forward coupling using Bragg reflectors [8431-17]

A. Lupu, Institut d'Électronique Fondamentale, CNRS, Univ. Paris-Sud (France); K. Muhieddine, E. Cassan, CNRS (France); J.-M. Lourtioz, Institut d'Électronique Fondamentale, CNRS, Univ. Paris-Sud (France)

\section{SILICON AND GERMANIUM MODULATOR}

8431 0J Ring resonator silicon optical modulator based on interleaved PN junctions [8431-18] M. Ziebell, D. Marris-Morini, G. Rasigade, Institut d'Electronique Fondamentale, CNRS, Univ. Paris-Sud (France); J.-M. Fédéli, CEA, LETI, Minatec (France); E. Cassan, L. Vivien, Institut d'Electronique Fondamentale, CNRS, Univ. Paris-Sud (France)

8431 OK Slow light enhanced carrier depletion modulators with IV drive voltage [8431-19] A. Brimont, Univ. Politécnica de Valencia (Spain); D. J. Thomson, Univ. of Southampton (United Kingdom); J. Herrera, Univ. Politècnica de València (Spain); F. Y. Gardes, Univ. of Southampton (United Kingdom); J. M. Fedeli, CEA, LETI, Minatec (France); G. T. Reed, CEA, LETI, Minatec (United Kingdom); J. Martí, P. Sanchis, Univ. Politècnica de València (Spain)

$8431 \mathrm{OM}$ The effects of strain on indirect absorption in Ge/SiGe quantum wells [8431-21] L. Lever, Z. Ikonić, R. W. Kelsall, Univ. of Leeds (United Kingdom) 
$8431 \mathrm{ON}$ Electroabsorption based on quantum-confined Stark effect from Ge/SiGe multiple quantum wells [8431-22]

P. Chaisakul, D. Marris-Morini, Institut d'Électronique Fondamentale, CNRS, Univ. Paris-Sud (France); G. Isella, D. Chrastina, Lab. for Epitaxial Nanostructures on Silicon and Spintronics (Italy); M.-S. Rouifed, Institut d'Électronique Fondamentale, CNRS, Univ. Paris-Sud (France); J. Frigerio, Lab. for Epitaxial Nanostructures on Silicon and Spintronics (Italy); E. Gatti, Univ. degli Studi di Milano-Bicocca (Italy); X. Le Roux, S. Edmond, E. Cassan, J.-R. Coudevylle,

L. Vivien, Institut d'Électronique Fondamentale, CNRS, Univ. Paris-Sud (France)

\section{CMOS PHOTONICS FOR THERANOSTICS}

843100 Real-time and low-cost biosensors based on photonic bandgap structures (Invited Paper) [8431-23]

J. García-Rupérez, J. G. Castelló, V. Toccafondo, P. Pérez-Millán, Univ. Politécnica de Valencia (Spain)

8431 OP Interferometric waveguide biosensors based on Si-technology for point-of-care diagnostic (Invited Paper) [8431-24]

D. Duval, A. B. González-Guerrero, S. Dante, CIN2 (Spain) and CIBER-BBN (Spain);

C. Domínguez, Ctr. Nacional de Microelectrónica (Spain); L. M. Lechuga, CIN2 (Spain) and CIBER-BBN (Spain)

$84310 Q \quad$ CMOS photonics for optical manipulation of particles and biosensing (Invited Paper) [8431-25]

A. W. Poon, H. Cai, Hong Kong Univ. of Science and Technology (Hong Kong, China)

8431 OS On-chip multiplexing concept for silicon photonic MZI biosensor array (Best Paper Award) [8431-27]

P. Muellner, R. Bruck, AlT Austrian Institute of Technology GmbH (Austria); M. Baus, M. Karl,

T. Wahlbrink, AMO GmbH (Germany); R. Hainberger, AlT Austrian Institute of Technology

$\mathrm{GmbH}$ (Austria)

\section{SILICON MODULATORS}

8431 OU CMOS-compatible electro-optical Mach-Zehnder modulator based on the amorphous silicon technology [8431-29]

S. Rao, Univ. degli Studi Mediterranea di Reggio Calabria (Italy); G. Coppola, M. A. Gioffrè, Institute for Microelectronics and Microsystems, CNR (Italy); F. G. Della Corte, Univ. degli Studi Mediterranea di Reggio Calabria (Italy) and Institute for Microelectronics and Microsystems, CNR (Italy)

8431 OV High-speed silicon optical modulator based on a PIPIN diode [8431-30]

M. Ziebell, D. Marris-Morini, G. Rasigade, Institut d'Electronique Fondamentale, CNRS, Univ. Paris-Sud (France); J.-M. Fédéli, CEA, LETI, Minatec (France); E. Cassan, Institut d'Electronique Fondamentale, CNRS, Univ. Paris-Sud (France); G.-H. Duan, III-V Lab (France); L. Vivien, Institut d'Electronique Fondamentale, CNRS, Univ. Paris-Sud (France)

8431 OW Low-voltage high-speed Si Mach-Zehnder optical modulator with multi-cascade $\mathrm{p} / \mathrm{n}$ junctions along waveguides [8431-31]

R. Furutani, Y. Amemiya, M. Fukuyama, S. Yokoyama, Hiroshima Univ. (Japan) 
843114 Co-integration of Ge detectors and Si modulators in an advanced Si photonics platform [8431-39]

P. Verheyen, G. Lepage, J. Van Campenhout, M. Pantouvaki, P. Absil, IMEC (Belgium);

P. De Heyn, W. Bogaerts, P. Dumon, S. Selvaraja, Ghent Univ. (Belgium)

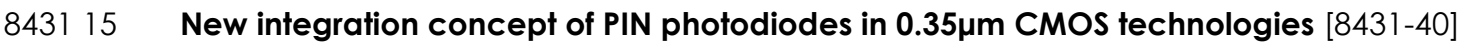

I. Jonak-Auer, J. Teva, J. M. Park, S. Jessenig, M. Rohrbacher, E. Wachmann,

austriamicrosystems AG (Austria)

\section{SOURCE ON SILICON}

843116 Er- and Nd-implanted MOS light emitting devices and their use for integrated photonic applications [8431-41]

L. Rebohle, R. Wutzler, S. Germer, J. Lehmann, M. Helm, W. Skorupa, Helmholtz-Zentrum

Dresden-Rossendorf e. V. (Germany)

843117 Carbon nanotube for photonics: light emission in silicon and optical gain [8431-42]

N. Izard, Institut d'Electronique Fondamentale, CNRS, Univ. Paris-Sud (France); E. Gaufrès, Institut d'Electronique Fondamentale, CNRS, Univ. Paris-Sud (France) and Univ. Montréal (Canada); A. Beck, A. Noury, X. Le Roux, L. Vivien, Institut d'Electronique Fondamentale, CNRS, Univ. Paris-Sud (France)

843118 Opto-electrical characterization of erbium-doped slot waveguides [8431-43]

A. Tengattini, D. Gandolfi, A. Marconi, A. Anopchenko, N. Prtljaga, Univ. degli Studi di Trento (Italy); J. M. Ramirez, F. Ferrarese Lupi, Y. Berencen, D. Navarro Urrios, B. Garrido, Univ. de Barcelona (Spain); J.-M. Fedeli, P. Rivallin, K. Surana, CEA-LETI-Minatec (France); L. Pavesi, Univ. degli Studi di Trento (Italy)

843119 Room temperature direct-gap electroluminescence in Ge/SiGe quantum well waveguides (Best Student Paper Award) [8431-44]

P. Chaisakul, D. Marris-Morini, Institut d'Électronique Fondamentale, CNRS, Univ. Paris-Sud (France); G. Isella, D. Chrastina, J. Frigerio, Lab. for Epitaxial Nanostructures on Silicon and Spintronics (Italy); M. S. Rouifed, N. Izard, X. Le Roux, S. Edmond, J.-R. Coudevylle, L. Vivien, Institut d'Électronique Fondamentale, CNRS, Univ. Paris-Sud (France)

8431 1B Optically active $\mu$-disks resonators-based sensor for refractive index variation detection [8431-46]

F. Ferrarese Lupi, MIND-IN2UB, Univ. de Barcelona (Spain); D. Navarro-Urrios, Catalan Institute of Nanotechnology, CIN2-CSIC (Spain); J. Rubio-Garcia, Institut de Recerca en Energia de Catalunya (Spain); J. Monserrat, C. Domínguez, Instituto de Microelectrònica de Barcelona-Ctr. Nacional de Microelectrónica (Spain); B. Garrido, MIND-IN2UB, Univ. de Barcelona (Spain)

\section{NON-SILICON INTEGRATED OPTICAL DEVICES}

8431 1C Sol-gel thin films for photonic application (Invited Paper) [8431-47]

J. J. Jasieniak, Commonwealth Scientific and Industrial Research Organisation (Australia);

A. Martucci, Univ. degli Studi di Padova (Italy) 
8431 ID Lithium niobate-on-insulator (LNOI): status and perspectives (Invited Paper) [8431-48] H. Hu, J. Yang, Shandong Univ. (China); L. Gui, W. Sohler, Univ. Paderborn (Germany)

8431 IF Erbium-activated silica-tin oxide glass ceramics for photonic integrated circuits: fabrication, characterisation, and assessment [8431-50]

T. T. V. Tran, LASIR, CNRS and CERLA, Univ. Lille 1 (France) and National Univ. of HoChiMinh (Viet Nam); C. Kinowski, O. Cristini, B. Capoen, PhLAM, CNRS and CERLA, Univ. Lille 1 (France); A. Chiasera, D. Ristic, M. Ferrari, Istituto di Fotonica e Nanotecnologie, CNR, CSMFO Lab. (Italy); S. Turrell, LASIR, CNRS and CERLA, Univ. Lille 1 (France)

8431 1G Tuning the emission wavelength of semiconductor ring lasers with on-chip filtered optical feedback [8431-51]

G. Verschaffelt, Vrije Univ. Brussel (Belgium); I. V. Ermakov, Univ. Catholique de Louvain (Belgium); S. Beri, Tyco Electronics (Belgium); M. Ashour, J. Danckaert, Vrije Univ. Brussel (Belgium); B. Docter, EFFECT Photonics BV (Netherlands); J. Bolk, X. Leijtens, Eindhoven Univ. of Technology (Netherlands)

$8431 \mathrm{lH} \quad$ High index contrast optical platform using gallium phosphide on sapphire: an alternative to sol? [8431-52]

D. O'Mahony, M. N. Hossain, J. Justice, E. Pelucchi, A. O'Riordan, B. Roycroft, B. Corbett, Tyndall National Institute (Ireland)

\section{PLASMONICS ON SILICON}

8431 11 Light propagation in metallic nanoparticle chains on SOI waveguide (Invited Paper) [8431-53]

M. Février, P. Gogol, A. Aassime, R. Megy, A. Bondi, Institut d'Électronique Fondamentale, CNRS, Univ. Paris-Sud (France) and Ctr. National de la Recherche Scientifique (France); A. Chelnokov, CEA-LETI-Minatec (France); A. Apuzzo, S. Blaize, G. Lerondel, LNIO, ICD, CNRS-FRE, Univ. de Technologie Troyes (France); J.-M. Lourtioz, B. Dagens, Institut d'Électronique Fondamentale, CNRS, Univ. Paris-Sud (France) and Ctr. National de la Recherche Scientifique (France)

$84311 \mathrm{~K}$ Proposal of a silicon optical modulator based on surface plasmon resonance [8431-55] T. Tabei, S. Yokoyama, Hiroshima Univ. (Japan)

\section{PASSIVE AND RESONANT PHOTONIC DEVICES}

8431 1M Low-nonlinearity and low-loss silicon slot waveguides with ALD-grown thin films [8431-57] L. Karvonen, A. Säynätjoki, Aalto Univ. (Finland); X. Tu, T. Y. Liow, A*STAR Institute of Microelectronics (Singapore); M. Hiltunen, J. Hiltunen, VTT Technical Research Ctr. of Finland (Finland); A. Tervonen, Aalto Univ. (Finland); G. Q. LO, A*STAR Institute of Microelectronics (Singapore); S. Honkanen, Aalto Univ. (Finland) and Univ. of Eastern Finland (Finland)

843110 Silicon-based monolithically integrated whispering-gallery mode resonators with buried waveguides [8431-59]

M. Ghulinyan, Fondazione Bruno Kessler (Italy); F. Ramiro Manzano, R. Guider, N. Prtljaga, Univ. of Trento (Italy); G. Pucker, Fondazione Bruno Kessler (Italy); L. Pavesi, Univ. of Trento (Italy) 
$8431 \mathrm{lP} \quad$ Whispering gallery modes in coated silica microspheres [8431-60]

D. Ristić, A. Chiasera, IFN-CNR, CSMFO Lab. (Italy); E. Moser, Trento Univ. (Italy); P. Féron, ENSSAT-FOTON, CNRS (France); G. Cibiel, Ctr. National d'Études Spatiales (France); M. Ivanda, Institut Ruder Boskovic (Croatia); G. C. Righini, IFAC-CNR, MDF Lab. (Italy); M. Ferrari, IFN-CNR, CSMFO Lab. (Italy)

\section{BIOSENSING}

$8431 \mathrm{IQ}$ A single-lithography SOI rib waveguide sensing circuit with apodized low back-reflection surface grating fiber coupling [8431-14]

V. J. Dubois, M. Antelius, H. Sohlström, K. B. Gylfason, Royal Institute of Technology (Sweden)

8431 IR Low-loss single mode light waveguides in polymer [8431-62]

H. Sieber, H.-J. Boehm, U. Hollenbach, J. Mohr, Karlsruhe Institute of Technology (Germany);

U. Ostrzinski, K. Pfeiffer, micro resist technology GmbH (Germany); M. Szczurowski,

W. Urbanczyk, Wroclaw Univ. of Technology (Poland)

8431 is Slot ring biosensors using silicon nitride waveguides with small temperature coefficient [8431-63]

A. Hirowatari, T. Taniguchi, T. Ikeda, M. Fukuyama, Y. Amemiya, A. Kuroda, S. Yokoyama, Hiroshima Univ. (Japan)

\section{POSTER SESSION}

8431 IT A diode-based bolometer implemented on micromachined CMOS technology for terahertz radiation detection [8431-64]

M. Perenzoni, S. Domingues, Fondazione Bruno Kessler (Italy)

$84311 \mathrm{U}$ Arrayed waveguide gratings beyond communication: utilization of entire image-plane of output star-coupler for spectroscopy and sensing [8431-65]

H. N. J. Fernando, A. Stoll, J. C. Boggio, R. Haynes, M. M. Roth, Leibniz-Institut für Astrophysik Potsdam (Germany)

8431 IV Simulation of polymeric integrated Young interferometer sensor [8431-66] M. Kusko, National Institute for Research and Development in Microtechnologies (Romania)

8431 1X Approximate model of a DBR/F-P laser based on Raman effect in a silicon-on-insulator rib waveguide [8431-68]

A. Tyszka-Zawadzka, Warsaw Univ. of Technology (Poland); P. Szczepański, Warsaw Univ. of Technology (Poland) and National Institute of Telecommunications (Poland); M. Karpierz, A. Mossakowska-Wyszyńska, M. Bugaj, Warsaw Univ. of Technology (Poland)

8431 1Y Effect of rapid thermal annealing on $\mathrm{Nd}^{-S i O_{x}}$ thin film properties [8431-69] C.-H. Liang, J. Cardin, L. Khomenkova, F. Gourbilleau, CIMAP (CNRS/CEA/ENSICAEN/UCBN) (France) 
843112 Quantum well intermixing in AllnGaAs QW structures through the interdiffusion of group III atoms [8431-70]

K.-H. Lee, K. Thomas, A. Gocalinska, M. Manganaro, H. Yang, E. Pelucchi, Tyndall National Institute (Ireland); F. H. Peters, Tyndall National Institute (Ireland) and Univ. College Cork (Ireland); B. Corbett, Tyndall National Institute (Ireland)

843121 A photo-sensor on thin polysilicon membrane embedded in wafer level package LED [8431-72]

J. K. Kim, H. C. Lee, KAIST (Korea, Republic of)

843122 Design of rare-earth doped chalcogenide microspheres for mid-IR optical amplification [8431-73]

F. Prudenzano, L. Mescia, P. Bia, A. Di Tommaso, M. De Sario, Politecnico di Bari (Italy); M. Ferrari, IFN-CNR, CSMFO Lab. (Italy); G. C. Righini, IFAC-CNR (Italy)

843123 Direct laser writing of 3D micro/nanostructures on opaque surfaces [8431-74]

S. Rekštytè, A. Žukauskas, V. Purlys, Vilnius Univ. (Lithuania); Y. Gordienko, G. V. Kurdyumov Institute for Metal Physics (Ukraine); M. Malinauskas, Vilnius Univ. (Lithuania)

843124 Improved integrated resonators in polymer technology for tunable filter [8431-75]

A. Maalouf, C. Bastianelli, Lab. Foton, CNRS, Univ. Européenne de Bretagne (France) and Ecole Nationale Supérieure des Sciences Appliquées et de Technologie (France); F. Mahé, Lab. IRMAR, CNRS, Univ. Européenne de Bretagne (France) and Univ. de Rennes (France); A. Belmiloudi, Lab. IRMAR, CNRS, Univ. Européenne de Bretagne (France) and INSA Rennes (France); M. Gadonna, Lab. Foton, CNRS, Univ. Européenne de Bretagne (France) and Télécom-Bretagne (France); D. Bosc, Lab. Foton, CNRS, Univ. Européenne de Bretagne (France) and Ecole Nationale Supérieure des Sciences Appliquées et de Technologie (France)

843126 A new type of sensitive semiconductor detectors of terahertz radiation [8431-77]

D. E. Dolzhenko, Lomonosov Moscow State Univ. (Russian Federation); A. V. Nicorici, Institute of Applied Physics (Moldova); L. I. Ryabova, D. R. Khokhlov, Lomonosov Moscow State Univ. (Russian Federation)

843127 High quality factor dielectric multilayer structures fabricated by rf-sputtering [8431-79] A. Chiasera, IFN-CNR, CSMFO Lab. (Italy); S. Valligatla, IFN-CNR, CSMFO Lab. (Italy), Univ. di Trento (Italy), and Univ. of Hyderabad (India); S. Varas, IFN-CNR, CSMFO Lab. (Italy); N. Bazzanella, Univ. di Trento (Italy); D. N. Rao, Univ. of Hyderabad (India); G. C. Righini, Ctr. Fermi (Italy) and IFAC-CNR (Italy); M. Ferrari, IFN-CNR, CSMFO Lab. (Italy)

843129 Low-frequency noise and microplasma measurements as a faster tool to investigate the quality of monocrystalline-silicon solar cells [8431-81]

Z. Chobola, M. Luňák, V. Juránková, J. Vanek, Brno Univ. of Technology (Czech Republic);

R. Barinka, Solartec, s.r.o. (Czech Republic)

Author Index 
Downloaded From: https://www.spiedigitallibrary.org/conference-proceedings-of-spie on 26 Apr 2023

Terms of Use: https://www.spiedigitallibrary.org/terms-of-use 


\title{
Conference Committee
}

\author{
Symposium Chairs
}

Francis Berghmans, Vrije Universiteit Brussel (Belgium)

Ronan Burgess, European Commission (Belgium)

Jürgen Popp, Institut für Photonische Technologien e.V. (Germany)

Peter Hartmann, SCHOTT AG (Germany)

Honorary Symposium Chair

Hugo Thienpont, Vrije Universiteit Brussel (Belgium)

\section{Conference Chairs}

Laurent Vivien, Institut d'Électronique Fondamentale, CNRS, Université Paris-Sud (France)

Seppo K. Honkanen, University of Eastern Finland (Finland)

Lorenzo Pavesi, Università degli Studi di Trento (Italy)

Stefano Pelli, Istituto di Fisica Applicata Nello Carrara (Italy)

Programme Committee

Vilson R. Almeida, Instituto de Estudos Avançados (Brazil)

Simona Binetti, Università degli Studi di Milano Bicocca (Italy)

Jean-Marc Fédéli, CEA-LETI (France)

Maurizio Ferrari, Istituto di Fotonica e Nanotecnologie (Italy)

Patrice Féron, Ecole Nationale Supérieure des Sciences Appliquées et de Technologie (France)

Michael Hochberg, University of Washington (United States)

Gong-Ru Lin, National Taiwan University (Taiwan)

Robert A. Norwood, College of Optical Sciences, The University of Arizona (United States)

Graham Trevor Reed, University of Surrey (United Kingdom)

Giancarlo C. Righini, Istituto di Fisica Applicata Nello Carrara (Italy)

Günther Roelkens, Universiteit Gent (Belgium)

Jung H. Shin, KAIST (Korea, Republic of)

Wolfgang Sohler, Universität Paderborn (Germany)

Jason Liow Tsung Yang, A*STAR Institute of Microelectronics

(Singapore)

Danxia Xu, National Research Council Canada (Canada)

Koji Yamada, Nippon Telegraph and Telephone Corporation (Japan)

YinZhong Yu, Institute of Semiconductors (China) 
Session Chairs

1 Nonlinear Integrated Photonics

Laurent Vivien, Institut d'Électronique Fondamentale, CNRS, Université Paris-Sud (France)

2 Nonlinear Optics

Lorenzo Pavesi, Università degli Studi di Trento (Italy)

3 Photodetectors

Seppo K. Honkanen, University of Eastern Finland (Finland)

$4 \quad$ Light Guiding and Coupling

Giancarlo C. Righini, Istituto di Fisica Applicata Nello Carrara (Italy)

$5 \quad$ Silicon and Germanium Modulator

Maria-Pilar Bernal, FEMTO-ST, CNRS, Université de Franche-Comté (France)

6 CMOS Photonics for Theranostics

Lorenzo Pavesi, Università degli Studi di Trento (Italy)

7 Silicon Modulators

Laurent Fulbert, CEA-LETI (France)

8 Industry and Silicon Photonics

Lorenzo Pavesi, Università degli Studi di Trento (Italy)

9 Silicon Photonics Integration

Bert-Jan Offrein, IBM Zürich Research Laboratory (Switzerland)

10 Source on Silicon

Kazumi Wada, The University of Tokyo (Japan)

11 Non-Silicon Integrated Optical Devices

Stefano Pelli, Istituto di Fisica Applicata Nello Carrara (Italy)

12 Plasmonics on Silicon

Seppo K. Honkanen, University of Eastern Finland (Finland)

13 Passive and Resonant Photonic Devices

Béatrice Dagens, Institut d'Électronique Fondamentale (France)

$14 \quad$ Biosensing

Laurent Vivien, Institut d'Électronique Fondamentale, CNRS, Université Paris-Sud (France) 


\section{Introduction}

This issue contains the papers presented at the third edition of the "Silicon Photonics and Photonic Integrated Circuits" Conference of Photonics Europe (2012). The conference was aimed to review and debate the hottest topics in integrated optical structures fabricated in silicon as well as in glasses, crystals, and polymers.

The conference was a success as was witnessed by the 81 submitted abstracts, a number which sharply rose above the 2010 edition. The conference success was also reflected in the high quality of the presentations, which in turn maintained the high interest of a large active audience.

The conference has shown that integrated optics, in particular silicon photonics, is able to provide technologies and devices suitable for industries or commercialization, while still keeping the style of a blue-sky research with the demonstration of interesting new phenomena. These new discoveries act as an innovation drive bound to be exploited by the industry in a longer time frame. This trend was particularly evident in the sessions devoted to sensing and to theranostics, where commercial achievements and novel approaches were concurrently illustrated.

The conference also caught a significant evolution of silicon photonics which moved from being a mostly datacom or interconnect oriented platform to a multipurpose platform for diverse applications, such as sensing, energetic, or medical applications.

This trend was evident in the four special sessions which covered:

- Nonlinear Integrated Photonics

- CMOS Photonics for Theranostics

- Industry and Silicon Photonics

- Non-Silicon Integrated Optical Devices.

We organized these special sessions mainly with invited presentations by experts who succeeded to efficiently highlight the most relevant research and industrial developments.

All sessions were well-attended, some exceptionally well, e.g., the one that focused on industry. They were all animated by many questions and comments from the participants, making them a valuable occasion for discussions and debates. 
The Best Student Paper Award offered by SPIE for this conference was presented to Papichaya Chaisakul, Institut d'Électronique Fondamentale, CNRS, Univ. ParisSud (France) for the paper on "Room temperature direct-gap electroluminescence in Ge/SiGe quantum well waveguides" (8431-44).

In cooperation with SPIE, we awarded three prizes to the best contributed paper presentations, which at the end of the conference we were delighted to confer to:

1) Carlos Alonso-Ramos, Univ. de Málaga (Spain) for the paper on "Grating couplers in thick rib SOI waveguides for TE and TM polarizations" (8431-13)

2) Paul Muellner, AIT Austrian Institute of Technology GmbH (Austria) for the paper on "On-chip multiplexing concept for silicon photonic MZI biosensor array" (8431-27)

3) Alexandros Emboras, CEA-LETI-Minatec (France) for the paper on "CMOS integration of field effect plasmonic modulator" (8431-56).

The good outcome of this conference has been a result of a cooperative work and we thank the members of the Programme Committee and the authors for this, and SPIE for the smooth organization of the symposium.

Laurent Vivien

Seppo K. Honkanen

Lorenzo Pavesi

Stefano Pelli 